\title{
Estimating Concrete Workability Based on Slump Test with Least Squares Support Vector Regression
}

\author{
Nhat-Duc Hoang' and Anh-Duc Pham ${ }^{2}$ \\ ${ }^{1}$ Institute of Research and Development, Faculty of Civil Engineering, Duy Tan University, \\ P809-K7/25 Quang Trung, Danang 550000, Vietnam \\ ${ }^{2}$ Faculty of Project Management, the University of Danang-University of Science and Technology, \\ 54 Nguyen Luong Bang, Danang 550000, Vietnam \\ Correspondence should be addressed to Nhat-Duc Hoang; hoangnhatduc@dtu.edu.vn
}

Received 23 August 2016; Accepted 8 November 2016

Academic Editor: Khandaker Hossain

Copyright (C) 2016 N.-D. Hoang and A.-D. Pham. This is an open access article distributed under the Creative Commons Attribution License, which permits unrestricted use, distribution, and reproduction in any medium, provided the original work is properly cited.

\begin{abstract}
Concrete workability, quantified by concrete slump, is an important property of a concrete mixture. Concrete slump is generally known to affect the consistency, flowability, pumpability, compactibility, and harshness of a concrete mix. Hence, an accurate prediction of this property is a practical need of construction engineers. This research proposes a machine learning model for predicting concrete slump based on the Least Squares Support Vector Regression (LS-SVR). LS-SVR is employed to model the nonlinear mapping between the mix components and slump values. Since the learning process of the LS-SVR necessitates two hyperparameters, the regularization and the kernel parameters, the grid search method is employed search for the most desirable set of hyperparameters. Furthermore, to construct the hybrid model, this research collected a dataset including actual concrete slump tests from a hydroelectric dam construction project in Vietnam. Experimental results show that the proposed model is capable of predicting concrete slump accurately.
\end{abstract}

\section{Introduction}

Concrete workability is defined as the effort required to manipulate a freshly mixed quantity of concrete with minimum loss of homogeneity [1]. This property of concrete is generally known to affect the consistency, flowability, pumpability, compactibility, and harshness of a concrete mix. Thus, concrete workability is a very crucial factor that must be considered in order to produce high quality concrete [2-4].

The slump test is the most common method for assessing the flow properties of fresh concrete; the slump provides a measure of workability [5]. Using this test, the slump can be derived by measuring the drop from the top of the slumped fresh concrete. In the task of concrete mixture design, the prediction of concrete flowability is critical for on-site construction. As the complexity of concrete construction escalates, there is an increasing pressure on material engineers to achieve high workability as well as to maintain the necessary mechanical properties to meet design specifications.

Concrete has been increasingly utilized in high-rise building and infrastructure development projects and special ingredients are often employed to make the material satisfy a specific set of performance requirements [6]. Superplasticizers are often included to enhance the concrete workability [7-9]. This situation makes the concrete mixes to be highly complex materials and modeling their properties becomes a very challenging task. There are complex and nonlinear relationships between the characteristics and the components that constitute the concrete mixes $[8,10,11]$.

Due to the importance of the research topic, various studies have been dedicated to concrete slump prediction. Traditional statistical models and machine learning are prevailing approaches to tackle the problem at hand. Öztaş et al. [2], Yeh [1, 3], Chine et al. [12], and Bilgil [13] employed the regression analysis and Artificial Neural Network (ANN) 

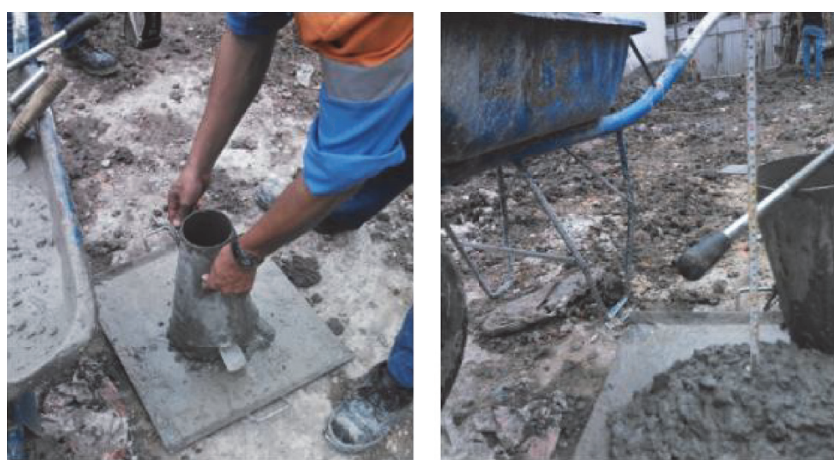

FIGURE 1: Concrete slump test (image source: http://xaydungthanhvinh.vn/).

models to estimate concrete slump; the common finding is that $\mathrm{ANN}$ is an effective nonlinear modeling method and its results are more accurate than the models based on the traditional regression analysis approach.

Baykasoğlu et al. [14] utilized the gene expression programming (GEP) to model high-strength concrete slump. Chen et al. [15] constructed a parallel hypercubic GEP to forecast the slump of high-performance concrete; this research showed that the improved method is better than the GEP and similar to the performance of ANN. Chandwani et al. [16] proposed a Genetic Algorithm assisted ANN; the study showed that the integrated approach can enhance the convergence speed of ANN and its prediction accuracy.

Due to the popularity of concrete in the construction industry, better alternatives for concrete slump prediction are of practical need for construction engineers in concrete mix design. This research contributes to the body of knowledge by proposing a new approach for improving the accuracy of concrete slump prediction which is based on the Least Squares Support Vector Regression (LS-SVR). LS-SVR is an advanced machine learning method which is designed for nonlinear modeling [17]; the superiority of the approach has been illustrated in recent applications [18-22].

Furthermore, a dataset that contains slump test records, collected from a hydroelectric dam construction project in central Vietnam, is used to establish and verify the proposed approach. The rest of the article is organized as follows: the second section presents the research method. The proposed slump prediction model is described in the third section. The next section reports the experimental results. The conclusion of this study is stated in the final section.

\section{Research Method}

2.1. The Concrete Slump Test Dataset. This research recorded testing results of 95 concrete mixes during the construction progress of the Song Bung 2 hydroelectric dam construction project in central Vietnam (http://www.sb2.vn/). The test is in conformity with the Vietnamese standard (TCVN-3106) for slump test which is equivalent to the ASTM-C-143. The equipment for the slump test includes a hollow frustum of a cone and a ruler as the measuring device (see Figure 1).
TABLE 1: Statistical description.

\begin{tabular}{lccccc}
\hline Factors & Notation & Min & Mean & Std. dev. & Max \\
\hline Cement $\left(\mathrm{kg} / \mathrm{m}^{3}\right)$ & $x_{1}$ & 201.0 & 338.6 & 62.3 & 446.3 \\
Natural sand $\left(\mathrm{kg} / \mathrm{m}^{3}\right)$ & $x_{2}$ & 384.0 & 690.1 & 154.2 & 827.0 \\
Crushed sand $\left(\mathrm{kg} / \mathrm{m}^{3}\right)$ & $x_{3}$ & 0.0 & 81.6 & 159.0 & 399.0 \\
Coarse aggregate $\left(\mathrm{kg} / \mathrm{m}^{3}\right)$ & $x_{4}$ & 1107.0 & 1155.7 & 33.0 & 1218.0 \\
Water (liter $\left./ \mathrm{m}^{3}\right)$ & $x_{5}$ & 164.0 & 174.2 & 6.4 & 186.0 \\
Superplasticizer $\left(\mathrm{liter} / \mathrm{m}^{3}\right)$ & $x_{6}$ & 1.0 & 3.1 & 0.9 & 4.5 \\
Concrete slump $(\mathrm{cm})$ & $y$ & 8.0 & 10.2 & 2.9 & 19.0 \\
\hline
\end{tabular}

TABLE 2: The dataset of concrete slump test.

\begin{tabular}{lccccccc}
\hline Mix & $x_{1}$ & $x_{2}$ & $x_{3}$ & $x_{4}$ & $x_{5}$ & $x_{6}$ & $y$ \\
\hline 1 & 261.0 & 397.0 & 397.0 & 1200.0 & 176.0 & 1.3 & 8.0 \\
2 & 248.0 & 399.0 & 399.0 & 1208.0 & 176.0 & 1.2 & 8.0 \\
3 & 320.0 & 388.0 & 388.0 & 1154.0 & 179.0 & 3.2 & 9.0 \\
4 & 304.0 & 391.0 & 391.0 & 1162.0 & 179.0 & 3.0 & 9.0 \\
5 & 336.0 & 385.0 & 385.0 & 1148.0 & 178.0 & 3.4 & 9.5 \\
6 & 356.0 & 769.0 & 0.0 & 1154.0 & 170.0 & 3.2 & 10.0 \\
$\vdots$ & $\vdots$ & $\vdots$ & $\vdots$ & $\vdots$ & $\vdots$ & $\vdots$ & $\vdots$ \\
90 & 339.0 & 765.0 & 0.0 & 1154.0 & 177.0 & 3.4 & 8.5 \\
91 & 375.0 & 770.0 & 0.0 & 1144.0 & 167.0 & 3.4 & 10.0 \\
92 & 393.0 & 757.0 & 0.0 & 1144.0 & 166.0 & 3.5 & 11.0 \\
93 & 404.0 & 767.0 & 0.0 & 1124.0 & 166.0 & 4.0 & 9.0 \\
94 & 378.0 & 751.0 & 0.0 & 1108.0 & 186.0 & 3.8 & 18.0 \\
95 & 359.1 & 765.0 & 0.0 & 1111.0 & 186.0 & 3.6 & 19.0 \\
\hline
\end{tabular}

The height of the cone is $30 \mathrm{~cm}$. The diameter of the top and bottom of the cone is $10 \mathrm{~cm}$ and $20 \mathrm{~cm}$, respectively. The cone is filled with fresh concrete and then lifted vertically. The height difference between the concrete and the cone is the slump value.

In this study, the concrete slump conditioning factors are selected based on reviewing previous works [1, 12, 16, 23] on slump flow modeling and the availability of measuring equipment. The amounts of cement $\left(\mathrm{kg} / \mathrm{m}^{3}\right)$, natural sand $\left(\mathrm{kg} / \mathrm{m}^{3}\right)$, crushed sand $\left(\mathrm{kg} / \mathrm{m}^{3}\right)$, coarse aggregate $\left(\mathrm{kg} / \mathrm{m}^{3}\right)$, water $\left(\right.$ liter $\left./ \mathrm{m}^{3}\right)$, and superplasticizer $\left(\right.$ liter $/ \mathrm{m}^{3}$ ) are $\mathrm{mix}$ ingredients. For each mix design, the slump value obtained from the actual slump test experiment is recorded. Statistical descriptions of all specimens are shown in Table 1. The whole dataset is partially described in Table 2. It is noted that the amounts of cement $\left(x_{1}\right)$, natural sand $\left(x_{2}\right)$, crushed sand $\left(x_{3}\right)$, coarse aggregate $\left(x_{4}\right)$, water $\left(x_{5}\right)$, and superplasticizer $\left(x_{6}\right)$ are used as input factors to predict the outputs which are the concrete slump $(y)$.

2.2. Least Squares Support Vector Regression (LS-SVR). LSSVR, proposed by Suykens et al. [17], is an advanced machine learning algorithm which is constructed on the principal of structural risk minimization. This approach has been proved to be very efficient in nonlinear modeling. Notably, 


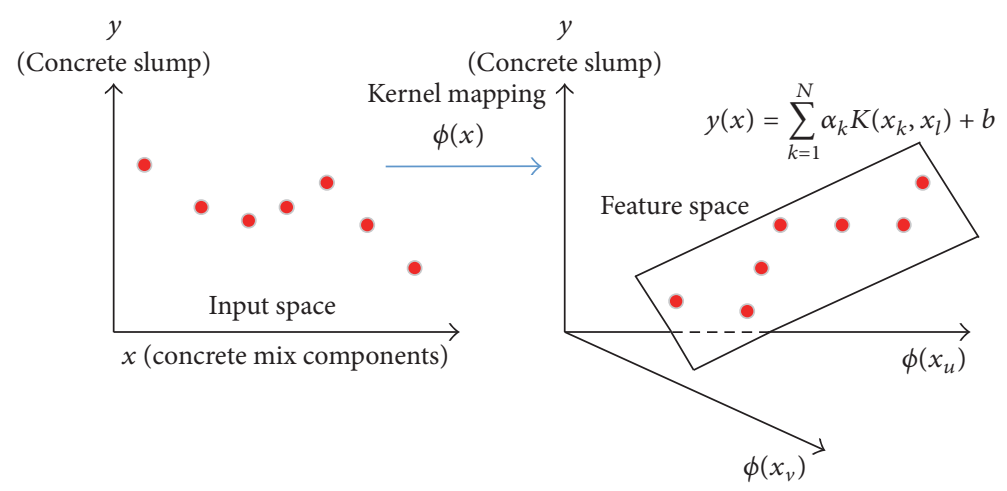

FIGURE 2: LS-SVR for concrete slump modeling.

the learning process of the LS-SVR is very fast since it only requires solving a set of linear equations.

To construct the prediction model, it is needed to prepare a dataset of slump test record in the form: $D=\left\{x_{k}, y_{k}\right\}$, $k=1,2, \ldots, N$. Herein, $k$ denotes the $k$ th data sample and $N$ is the total number of data samples. It is noted that $x_{k}$ is a vector with six elements; $x_{k 1}, x_{k 2}, x_{k 3}, x_{k 4}, x_{k 5}$, and $x_{k 6}$ denote the amount of cement, natural sand, crushed sand, coarse aggregate, water, and superplasticizer, respectively. Meanwhile, $y_{k}$ is the output of concrete slump of the $k$ th data sample.

We aim to establish a mapping function $y(x)$ that derives the output of concrete slump based on the input vector $x$ that describes the concrete mix components. Since the functional mapping between concrete mix components $(x)$ and slump value $(y)$ is possibly nonlinear, LS-SVR first maps the data from the original input space to a high-dimensional feature space via a mapping function $\phi(x)$. Accordingly, linear regression analysis can be possibly performed in such high-dimensional feature space. The operation of LS-SVR in concrete slump modeling is illustrated in Figure 2.

In the training phase of LS-SVR, the learning objective can be formulated as the following optimization problem [17, 24]:

$$
\text { Minimize } \quad J_{p}(w, e)=\frac{1}{2} w^{T} w+\gamma \frac{1}{2} \sum_{k=1}^{N} e_{k}^{2}
$$

Subjected to $y_{k}=w^{T} \phi\left(x_{k}\right)+b+e_{k}, \quad k=1, \ldots, N$,

where $e_{k} \in R$ are error variables; $\gamma>0$ denotes a regularization constant.

In order to solve the above optimization problem, the Lagrangian function is formulated as [17]

$$
\begin{aligned}
L(w, b, e ; \alpha)= & J_{p}(w, e) \\
& -\sum_{k=1}^{N} \alpha_{k}\left\{w^{T} \phi\left(x_{k}\right)+b+e_{k}-y_{k}\right\},
\end{aligned}
$$

where $\alpha_{k}$ are Lagrange multipliers.
The Karush-Kuhn-Tucker conditions for optimality are used by differentiating the Lagrangian function $L(w, b, e, \alpha)$ with the variables as follows [17]:

$$
\begin{aligned}
\frac{\partial L}{\partial w} & =0 \longrightarrow \\
w & =\sum_{k=1}^{N} \alpha_{k} \phi\left(x_{k}\right), \\
\frac{\partial L}{\partial b} & =0 \longrightarrow \\
\sum_{k=1}^{N} \alpha_{k} & =0, \\
\frac{\partial L}{\partial e_{k}} & =0 \longrightarrow \\
\alpha_{k} & =\gamma e_{k}, \quad k=1, \ldots, N, \\
\frac{\partial L}{\partial \alpha_{k}} & =0 \longrightarrow \\
w^{T} \phi\left(x_{k}\right)+b+e_{k}-y_{k} & =0, \quad k=1, \ldots, N .
\end{aligned}
$$

By solving linear system (3), the resulting LS-SVR model is expressed as follows $[17,18]$ :

$$
y(x)=\sum_{k=1}^{N} \alpha_{k} K\left(x_{k}, x_{l}\right)+b
$$

where $\alpha_{k}$ and $b$ are the solution to the linear system. $k$ and $N$ are the index and the total number of data points in the training set. $x_{k}$ and $x_{l}$ denote an input pattern in the training and testing set. It is worth reminding that $x_{k}$ and $x_{l}$ are both input vectors of concrete mix components with six elements. $K(\cdot)$ is the kernel function which maps the input data from 


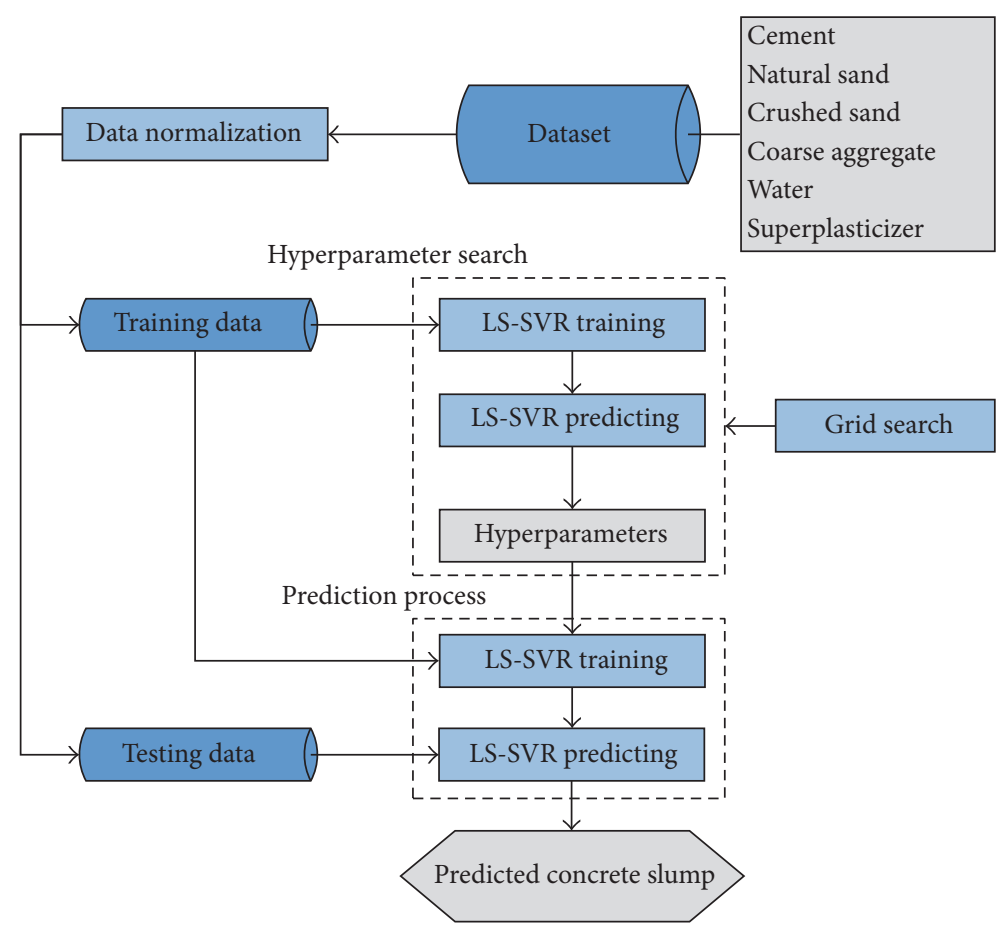

FIGURE 3: Concrete slump prediction using LS-SVR (CSP-LSSVR).

the feature space into the high-dimensional space. The radial basis kernel function is often employed $[17,19]$ :

$$
K\left(x_{k}, x_{l}\right)=\exp \left(-\frac{\left\|x_{k}-x_{l}\right\|^{2}}{2 \sigma^{2}}\right),
$$

where $\sigma$ represents the radial basis kernel function parameter.

\section{The Proposed Model for Concrete Slump Prediction}

This section of the article describes the concrete slump prediction using LS-SVR (CSP-LSSVR). The prediction model relies on LS-SVR to discover the nonlinear mapping relationship between the concrete components and the slump. The flowchart of the CSP-LSSVR is demonstrated in Figure 3.

Given the input data of concrete mix ingredients (the amounts of cement, natural sand, crushed sand, coarse aggregate, water, and superplasticizer), the first step of the model is to carry out the data normalization process within which the whole data is normalized into a $(0,1)$ range. This process can help prevent the circumstance in which inputs with greater magnitudes dominate those with smaller magnitudes. The function used for normalizing data is provided as follows:

$$
X_{n}=\frac{X_{o}-X_{\min }}{X_{\max }-X_{\min }},
$$

where $X_{n}$ is the normalized data. $X_{o}$ is the original data. $X_{\max }$ and $X_{\min }$ denote the maximum and minimum values of the data, respectively.
The dataset, featuring six input factors and the output variable of concrete slump, is then randomly divided into a training set and a testing set. The training dataset is employed to establish the LS-SVR model. Since the LS-SVR with radial basis kernel function is employed, the learning process requires hyperparameters, the regularization parameter $\gamma$ and the kernel parameter $\sigma$, and the grid search method $[17,25]$ is employed search for the most desirable set of hyperparameters.

In the grid search for tuning parameters, various pairs of ( $\gamma$ and $\delta$ ) are tried and the one with the best fivefold crossvalidation accuracy is chosen. Using exponential growing sequences of $\gamma\left(2^{-5}, 2^{-4}, \ldots, 2^{15}\right)$ and $\sigma\left(2^{-15}, 2^{-4}, \ldots, 2^{3}\right)$ is a common way to identify good parameters. The grid search approach is straightforward and easy to implement. After the hyperparameters have been determined appropriately and the training process is finished, the proposed CSP-LSSVR can be used to predict the slump flow values of new concrete samples.

\section{Experimental Results}

When the training process finishes, the slump of concrete mix in the testing cases can be predicted by providing mixture components for the trained model. In the experiments, besides the proposed CSP-LSSVR, the Artificial Neural Network (ANN) and the multiple linear regression (MLR) are utilized as benchmark methods. In order to measure model performance, this research employs Root Mean Squared 


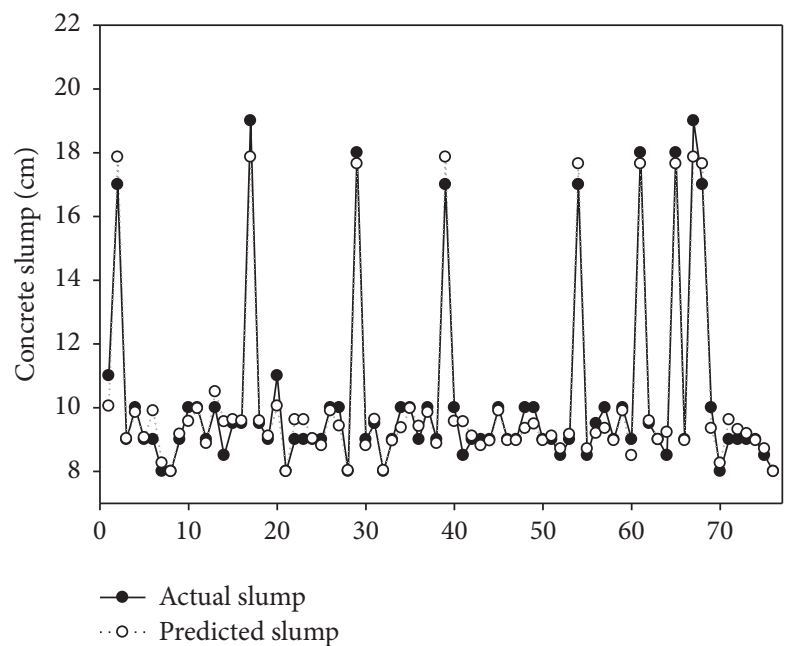

FIGURE 4: The CSP-LSSVR training results.

Error (RMSE), Mean Absolute Percentage Error (MAPE), and Coefficient of Determination $\left(R^{2}\right)$.

The motivation for using these benchmark approaches is that the ANN is an effective tool for nonlinear modeling and has been successfully employed for predicting concrete slump $[3,12,23]$. The MLR model is a basic statistical predictive method and comparing its result with other machine learning models may reveal useful insights [26].

To construct an ANN, the user needs to specify the network structure and the learning rate. Such parameters of the ANN model are usually selected via a trial-and-error process [26]. Based on experiments, the network configuration is set as follows: the number of hidden layers is set to be 1 ; the learning rate is 0.001 ; the number of neurons in the hidden layer is set to be 6. The Levenberg-Marquardt algorithm [27] is employed to train the ANN model.

In the first experiment, the dataset is randomly divided into 2 sets: the training set that occupies $80 \%$ of the dataset and the testing set that includes $20 \%$ of the dataset. In detail, the training and testing sets consist of 76 and 19 mixes, respectively. The training and testing results of the CSPLSSVR are illustrated in Figures 4 and 5, respectively.

The MLR model for predicting concrete slump based on the collected dataset is established via the Least Squares Estimation method [28] and shown as follows:

$$
\begin{aligned}
y= & 36.22-12.47 x_{1}-27.03 x_{2}-24.56 x_{3}-7.39 x_{4} \\
& -3.00 x_{5}-1.18 x_{6},
\end{aligned}
$$

where the symbols of $x_{1}, x_{2}, x_{3}, x_{4}, x_{5}$, and $x_{6}$ represent the amount of cement, natural sand, crushed sand, coarse aggregate, water, and superplasticizer within the concrete mix, respectively.

The ANN model structure, which contains the input, hidden, and output layers, is illustrated in Figure 6. It is noted that $W_{1}$ and $W_{2}$ are the weight matrices of the hidden layer and the output layer, respectively; $\Theta=6$ denotes the number of neurons in the hidden layer; $b_{1}=\left[b_{11}, b_{12}, \ldots, b_{1 \Theta}\right]$

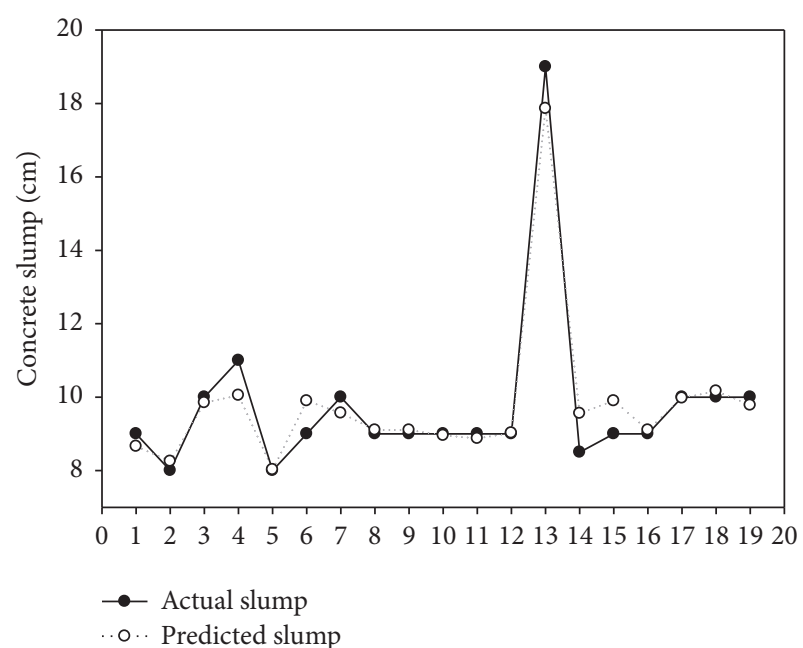

Figure 5: The CSP-LSSVR testing results.

represents the bias vector of the hidden layer; $b_{2}$ denotes the bias vector of the output layer; $n_{i}$ is the output of the $i$ th neuron in the hidden layer; $F$ is the tan-sigmoid activation function which is commonly used in the hidden layer $[29,30]$ :

$$
f_{A}(v)=\frac{1}{1+\exp (-v)},
$$

where $v$ denotes an input for the function.

It is noted that the weight matrices $\left(W_{1}\right.$ and $\left.W_{2}\right)$ and the bias vectors $\left(b_{1}\right.$ and $b_{2}$ ) of the ANN model for concrete slump estimation are learnt via a training process with the error backpropagation algorithm [31]. After the training phase, results of the ANN parameters are shown as follows:

$$
\begin{aligned}
& W_{1} \\
& =\left[\begin{array}{cccccc}
-1.4769 & 0.7164 & -1.2991 & -2.4781 & -0.1608 & -1.7888 \\
-2.4336 & -0.5163 & 0.0260 & 3.3005 & -2.5351 & -0.3737 \\
-2.6319 & 0.2066 & 2.9821 & -2.0341 & -4.2239 & 0.1737 \\
1.0796 & 0.1646 & -0.6626 & -1.8433 & -0.0726 & -5.0730 \\
2.7421 & -1.1328 & 0.0858 & -3.7346 & 3.4695 & 1.7114 \\
-0.8771 & 0.3779 & 0.5195 & -2.5811 & -0.7168 & -3.2345
\end{array}\right], \\
& W_{2}=\left[\begin{array}{llllll}
1.9870 & 0.5667 & 1.3222 & 2.4838 & 0.5420 & -3.5485
\end{array}\right], \\
& b_{1}=\left[\begin{array}{llllll}
-2.6084 & 1.4889 & -0.3951 & 0.2309 & -1.1501 & 0.3311
\end{array}\right], \\
& b_{2}=0.7132 .
\end{aligned}
$$

Table 3 provides the result comparison between the proposed method and other benchmark models. The result of the MLR in the testing process is very poor (RMSE = 0.28, MAPE $=12.08 \%, R^{2}=0.28$; this indicates that the linear model is insufficient to explain the behavior of concrete slump.

The ANN and CSP-LSSVR models achieve much better performances; both models have the $R^{2}$ values which are 


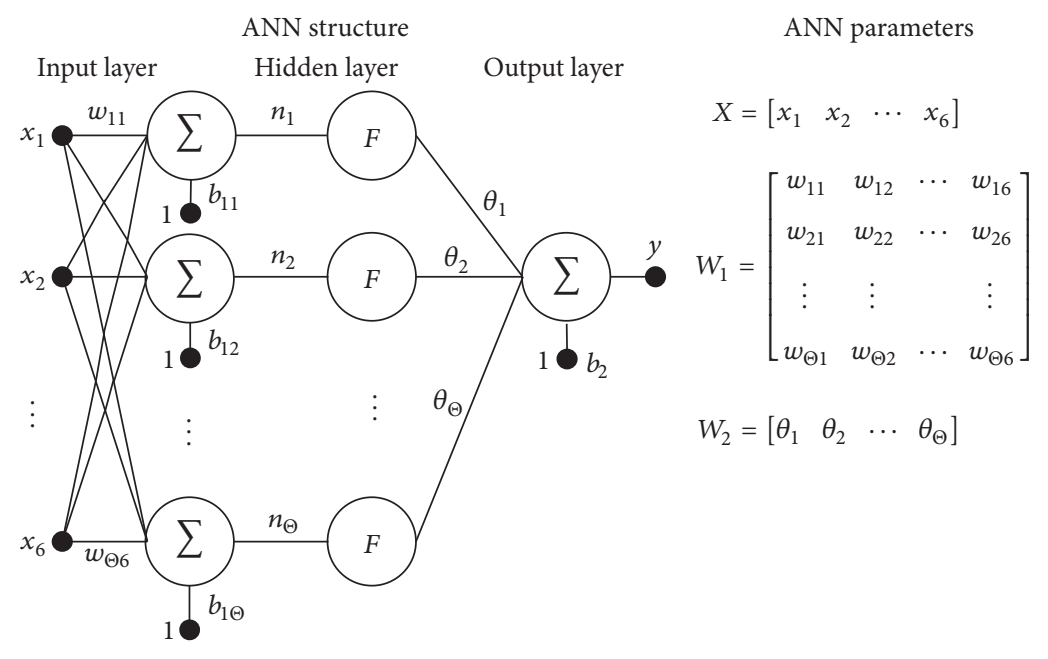

FIgURE 6: The ANN model structure.

TABLE 3: Result comparison.

\begin{tabular}{lccc}
\hline & MLR & ANN & CSP-LSSVR \\
\hline Training & & & \\
RMSE & 1.23 & 0.94 & 0.46 \\
MAPE (\%) & 9.62 & 3.63 & 3.07 \\
$R^{2}$ & 0.82 & 0.91 & 0.97 \\
Testing & & & \\
RMSE & 1.54 & 1.05 & 0.54 \\
MAPE (\%) & 12.08 & 5.96 & 3.68 \\
$R^{2}$ & 0.28 & 0.83 & 0.96 \\
\hline
\end{tabular}

greater than 0.8. According to Smith [32], such high values of $R^{2}$ imply strong correlations between the predicted and measured concrete slumps. Furthermore, the CSP-LSSVR has achieved the lowest prediction error $(\mathrm{MAPE}=3.68 \%$ and RMSE = 0.54). Thus, benchmarked with the ANN, the new method has attained $38 \%$ and $49 \%$ reductions in terms of MAPE and RMSE.

Moreover, to avoid the randomness in selecting testing samples, the second experiment carries out a 10-fold crossvalidation process. Using the cross-validation process, the whole dataset is randomly divided into 10 data folds in which each fold in turn serves as a testing set; and the performance of the model can be assessed by averaging results of the 10 folds. Because all of the subsamples are mutually exclusive, this experiment can evaluate the CSPLSSVR more accurately.

Table 4 summarizes the result of the cross-validation process. Observably, the proposed approach has attained the lowest prediction error in both training and testing processes. The average RMSE and MAPE for testing data of the CSPLSSVR are 0.50 and $2.81 \%$, respectively. These prediction errors are significantly lower than the ANN (RMSE $=0.62$ and $4.44 \%$ ) and the MLR (RMSE $=1.36$ and 10.64\%). The proposed approach also yields the highest $R^{2}(0.90)$ when predicting the slump of testing concrete mixes. Hence, the experimental results have strongly demonstrated the superior predictive capability of the CSP-LSSVR model.

\section{Conclusion}

This study has established a new method for predicting concrete workability quantified by the slump values. The research extends the body of knowledge by investigating the capability of LS-SVR for concrete slump prediction. To establish the proposed CSP-LSSVR, a dataset consisting of actual concrete slump tests has been collected. From the experiments, the proposed model has achieved the most accurate prediction results.

The average MAPE of the method obtained from the cross-validation process is less than $3 \%$ which is very desirable because modeling concrete slump is known to be very complex and highly nonlinear. Since the tenfold crossvalidation process is a very reliable way for model performance evaluation [33], it is expected that the proposed CSPLSSVR can predict the flow of concrete based on the similar conditioning factors with the same accuracy. Accordingly, the newly established method can be a very useful tool to assist the engineers in the task of concrete mix design.

Nevertheless, in addition to the currently used six conditioning factors of concrete slump, other factors (e.g., the type, size, absorption, and the water amount of the fine and coarse aggregates) can be relevant and should be considered by the model. Furthermore, another limitation of the current study is that the employed dataset only consists of 95 data points. Thus, this dataset should be expanded in a future study to further enhance the generalization of the current model and better ensure the predictive accuracy of the model when dealing with new concrete mixes. 
TABLE 4: The result of the 10-fold cross-validation process.

\begin{tabular}{|c|c|c|c|c|c|c|c|c|c|c|c|c|}
\hline \multicolumn{2}{|c|}{ Data fold } & 1 & 2 & 3 & 4 & 5 & 6 & 7 & 8 & 9 & 10 & Avg. \\
\hline \multirow{8}{*}{ CSP-LSSVR } & Training & & & & & & & & & & & \\
\hline & RMSE & 0.46 & 0.32 & 0.24 & 0.30 & 0.25 & 0.29 & 0.29 & 0.26 & 0.69 & 0.29 & 0.34 \\
\hline & MAPE & 3.15 & 1.37 & 0.65 & 1.13 & 0.68 & 0.90 & 0.88 & 0.96 & 5.21 & 0.92 & 1.59 \\
\hline & $R^{2}$ & 0.97 & 0.99 & 0.99 & 0.99 & 0.99 & 0.99 & 0.99 & 0.99 & 0.94 & 0.99 & 0.98 \\
\hline & Testing & & & & & & & & & & & \\
\hline & RMSE & 0.68 & 0.33 & 0.64 & 0.46 & 0.58 & 0.39 & 0.38 & 0.67 & 0.49 & 0.37 & 0.50 \\
\hline & MAPE & 4.50 & 2.29 & 2.22 & 2.60 & 2.69 & 1.70 & 2.16 & 3.51 & 3.92 & 2.52 & 2.81 \\
\hline & $R^{2}$ & 0.99 & 0.83 & 1.00 & 0.58 & 0.98 & 0.99 & 0.99 & 0.97 & 0.97 & 0.72 & 0.90 \\
\hline \multirow{8}{*}{ ANN } & Training & & & & & & & & & & & \\
\hline & RMSE & 0.56 & 0.47 & 0.48 & 0.74 & 0.57 & 0.61 & 0.36 & 0.50 & 0.44 & 0.46 & 0.52 \\
\hline & MAPE & 4.27 & 3.13 & 3.45 & 3.00 & 3.78 & 4.25 & 1.56 & 3.43 & 2.56 & 2.36 & 3.18 \\
\hline & $R^{2}$ & 0.96 & 0.97 & 0.96 & 0.94 & 0.96 & 0.96 & 0.98 & 0.97 & 0.98 & 0.97 & 0.97 \\
\hline & Testing & & & & & & & & & & & \\
\hline & RMSE & 0.62 & 0.53 & 0.71 & 1.10 & 0.51 & 0.58 & 0.40 & 0.83 & 0.31 & 0.63 & 0.62 \\
\hline & MAPE & 4.97 & 5.21 & 4.33 & 6.30 & 3.58 & 4.11 & 2.38 & 6.68 & 1.65 & 5.17 & 4.44 \\
\hline & $R^{2}$ & 0.98 & 0.60 & 0.99 & 0.09 & 0.97 & 0.98 & 0.98 & 0.91 & 0.99 & 0.29 & 0.78 \\
\hline \multirow{8}{*}{ MLR } & Training & & & & & & & & & & & \\
\hline & RMSE & 1.23 & 1.25 & 1.19 & 1.20 & 1.27 & 1.21 & 1.25 & 1.27 & 1.29 & 1.23 & 1.24 \\
\hline & MAPE & 9.26 & 9.56 & 9.07 & 9.29 & 9.73 & 9.16 & 9.80 & 9.85 & 10.12 & 9.62 & 9.55 \\
\hline & $R^{2}$ & 0.79 & 0.81 & 0.75 & 0.83 & 0.80 & 0.79 & 0.79 & 0.79 & 0.79 & 0.82 & 0.80 \\
\hline & Testing & & & & & & & & & & & \\
\hline & RMSE & 1.56 & 1.28 & 1.78 & 1.67 & 1.09 & 1.58 & 1.28 & 1.04 & 0.78 & 1.54 & 1.36 \\
\hline & MAPE & 11.56 & 11.10 & 11.59 & 11.93 & 9.44 & 14.40 & 10.53 & 8.10 & 5.67 & 12.08 & 10.64 \\
\hline & $R^{2}$ & 0.90 & 0.01 & 0.91 & 0.01 & 0.80 & 0.82 & 0.79 & 0.85 & 0.90 & 0.28 & 0.63 \\
\hline
\end{tabular}

Note: Avg. denotes the average result.

\section{Competing Interests}

The authors declare that there is no conflict of interests regarding the publication of this manuscript.

\section{References}

[1] I.-C. Yeh, "Modeling slump flow of concrete using secondorder regressions and artificial neural networks," Cement and Concrete Composites, vol. 29, no. 6, pp. 474-480, 2007.

[2] A. Öztaş, M. Pala, E. Özbay, E. Kanca, N. Çağlar, and M. A. Bhatti, "Predicting the compressive strength and slump of high strength concrete using neural network," Construction and Building Materials, vol. 20, no. 9, pp. 769-775, 2006.

[3] I.-C. Yeh, "Exploring concrete slump model using artificial neural networks," Journal of Computing in Civil Engineering, vol. 20, no. 3, pp. 217-221, 2006.

[4] Y. Li, J. Wang, and Z. Xu, "Design optimization of a concrete face rock-fill dam by using genetic algorithm," Mathematical Problems in Engineering, vol. 2016, Article ID 4971048, 11 pages, 2016.

[5] P. K. Mehta and P. J. M. Monteiro, Concrete-Structure, Properties, and Materials, Prentice Hall Inc, Englewood Cliffs, NJ, USA, 1993.

[6] Y. Peng, H. Chu, and J. Pu, "Numerical simulation of recycled concrete using convex aggregate model and base force element method," Advances in Materials Science and Engineering, vol. 2016, Article ID 5075109, 10 pages, 2016.

[7] J. Kasperkiewicz, J. Racz, and A. Dubrawski, "HPC strength prediction using artificial neural network," Journal of Computing in Civil Engineering, vol. 9, no. 4, pp. 279-284, 1995.

[8] I.-C. Yeh, "Modeling of strength of high-performance concrete using artificial neural networks," Cement and Concrete Research, vol. 28, no. 12, pp. 1797-1808, 1998.

[9] S. U. Khan, M. F. Nuruddin, T. Ayub, and N. Shafiq, "Effects of different mineral admixtures on the properties of fresh concrete," The Scientific World Journal, vol. 2014, Article ID 986567, 11 pages, 2014.

[10] J.-S. Chou, C.-F. Tsai, A.-D. Pham, and Y.-H. Lu, "Machine learning in concrete strength simulations: multi-nation data analytics," Construction and Building Materials, vol. 73, pp. 771780, 2014.

[11] N. Hoang, A. Pham, Q. Nguyen, and Q. Pham, "Estimating compressive strength of high performance concrete with gaussian process regression model," Advances in Civil Engineering, vol. 2016, Article ID 2861380, 8 pages, 2016.

[12] W.-H. Chine, H.-H. Hsu, L. Chen, T.-S. Wang, and C.-H. Chiu, "Modeling slump of concrete using the artificial neural networks," in Proceedings of the International Conference on Artificial Intelligence and Computational Intelligence (AICI '10), pp. 236-239, Sanya, China, October 2010.

[13] A. Bilgil, "Estimation of slump value and Bingham parameters of fresh concrete mixture composition with artificial neural 
network modelling," Scientific Research and Essays, vol. 6, no. 8, pp. 1753-1765, 2011.

[14] A. Baykasoğlu, A. Öztaş, and E. Özbay, "Prediction and multiobjective optimization of high-strength concrete parameters via soft computing approaches," Expert Systems with Applications, vol. 36, no. 3, pp. 6145-6155, 2009.

[15] L. Chen, C.-H. Kou, and S.-W. Ma, "Prediction of slump flow of high-performance concrete via parallel hyper-cubic geneexpression programming," Engineering Applications of Artificial Intelligence, vol. 34, pp. 66-74, 2014.

[16] V. Chandwani, V. Agrawal, and R. Nagar, "Modeling slump of ready mix concrete using genetic algorithms assisted training of Artificial Neural Networks," Expert Systems with Applications, vol. 42, no. 2, pp. 885-893, 2015.

[17] J. Suykens, J. V. Gestel, J. D. Brabanter, B. D. Moor, and J. Vandewalle, Least Square Support Vector Machines, World Scientific, Singapore, 2002.

[18] J. Ji, C. Zhang, Y. Gui, Q. Lü, and J. Kodikara, "New observations on the application of LS-SVM in slope system reliability analysis," Journal of Computing in Civil Engineering, 2016.

[19] D. Yao, J. Yang, X. Li, and C. Zhao, "A hybrid approach for fault diagnosis of railway rolling bearings using STWD-EMDGA-LSSVM," Mathematical Problems in Engineering, vol. 2016, Article ID 8702970, 7 pages, 2016.

[20] J.-S. Chou and A.-D. Pham, "Smart artificial firefly colony algorithm-based support vector regression for enhanced forecasting in civil engineering," Computer-Aided Civil and Infrastructure Engineering, vol. 30, no. 9, pp. 715-732, 2015.

[21] D. Tien Bui, B. T. Pham, Q. P. Nguyen, and N. Hoang, "Spatial prediction of rainfall-induced shallow landslides using hybrid integration approach of Least-Squares Support Vector Machines and differential evolution optimization: a case study in Central Vietnam," International Journal of Digital Earth, vol. 9, no. 11, pp. 1077-1097, 2016.

[22] D.-T. Vu and N.-D. Hoang, "Punching shear capacity estimation of FRP-reinforced concrete slabs using a hybrid machine learning approach," Structure and Infrastructure Engineering, vol. 12, no. 9, pp. 1153-1161, 2016.

[23] V. Chandwani, V. Agrawal, and R. Nagar, "Modeling slump of ready mix concrete using genetically evolved artificial neural networks," Advances in Artificial Neural Systems, vol. 2014, Article ID 629137, 9 pages, 2014.

[24] M.-Y. Cheng and N.-D. Hoang, "Interval estimation of construction cost at completion using least squares support vector machine," Journal of Civil Engineering and Management, vol. 20, no. 2, pp. 223-236, 2014.

[25] C. W. Hsu, C. C. Chang, and C. J. Lin, "A practical guide to support vector classification," Tech. Rep., Department of Computer Science, National Taiwan University, Taipei, Taiwan, 2010.

[26] J.-S. Chou, C.-K. Chiu, M. Farfoura, and I. Al-Taharwa, "Optimizing the prediction accuracy of concrete compressive strength based on a comparison of data-mining techniques," Journal of Computing in Civil Engineering, vol. 25, no. 3, pp. 242253, 2011.

[27] M. T. Hagan and M. B. Menhaj, "Training feedforward networks with the Marquardt algorithm," IEEE Transactions on Neural Networks, vol. 5, no. 6, pp. 989-993, 1994.

[28] N. R. Draper and H. Smith, Applied Regression Analysis, WileyInterscience, 1998.

[29] M. T. Hagan, H. B. Demuth, and M. H. Beale, Neural Network Design, PWS Publishing, Boston, Mass, USA, 1996.
[30] T. Tran and N. Hoang, "Predicting colonization growth of algae on mortar surface with artificial neural network," Journal of Computing in Civil Engineering, vol. 30, no. 6, Article ID 04016030, 2016.

[31] D. E. Rumelhart, G. E. Hinton, and R. J. Williams, "Learning representations by back-propagating errors," Nature, vol. 323, no. 6088, pp. 533-536, 1986.

[32] G. N. Smith, Probability and Statistics in Civil Engineering, Collins, London, UK, 1986.

[33] S. Arlot and A. Celisse, "A survey of cross-validation procedures for model selection," Statistics Surveys, vol. 4, pp. 40-79, 2010. 


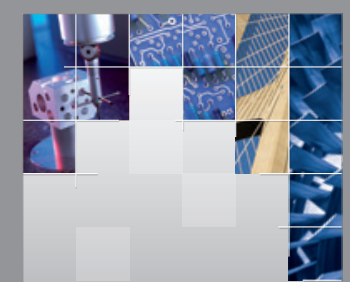

\section{Enfincering}
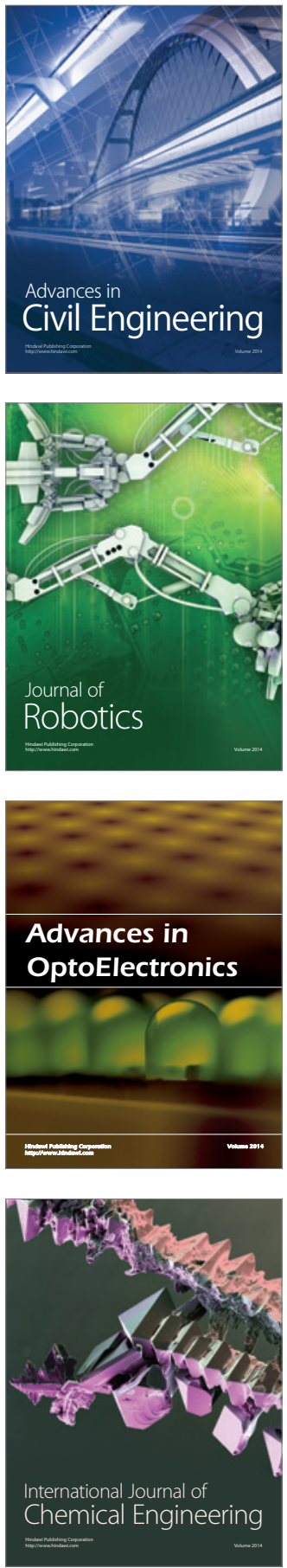

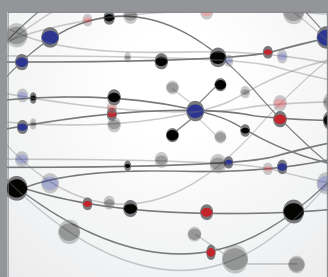

The Scientific World Journal

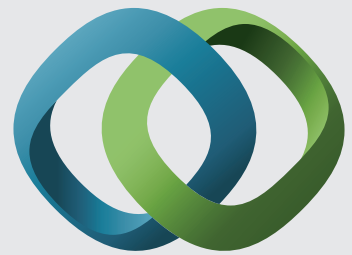

\section{Hindawi}

Submit your manuscripts at

http://www.hindawi.com
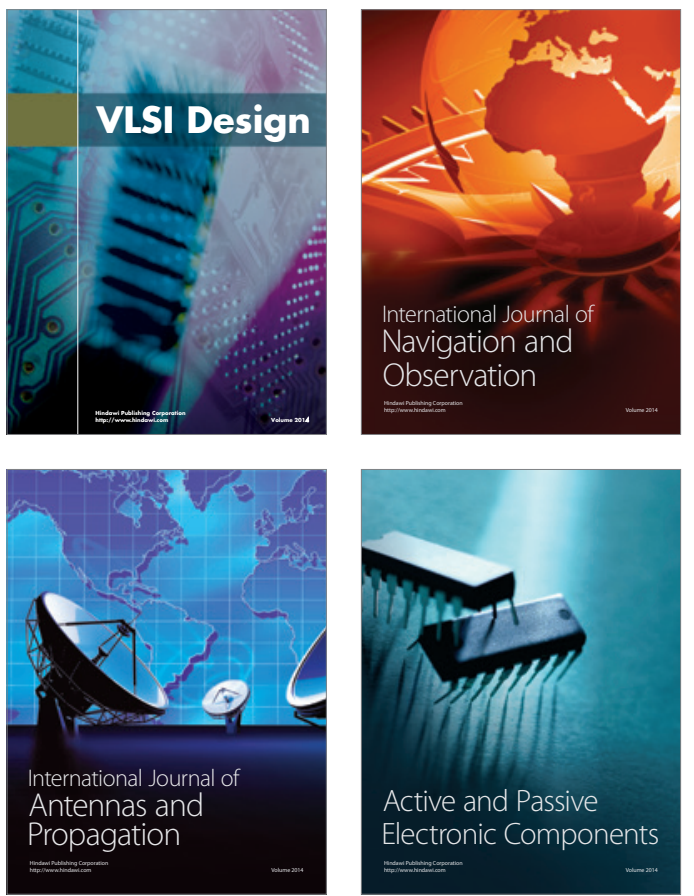
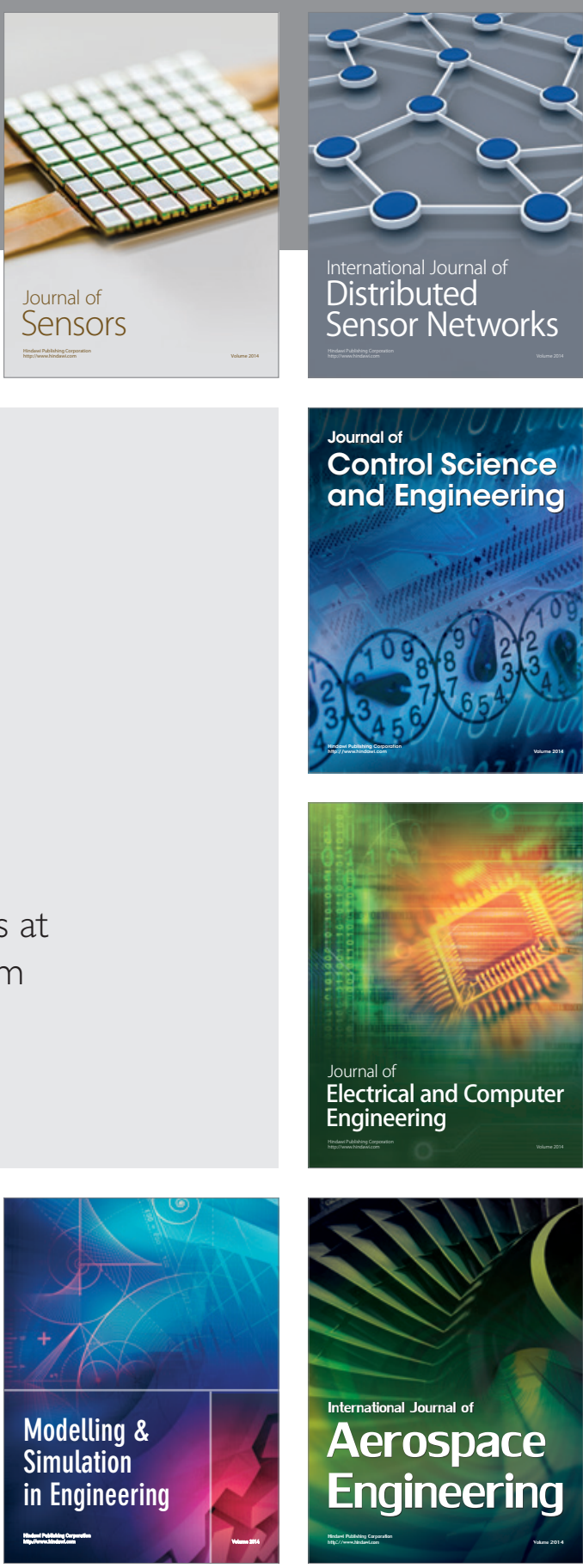

International Journal of

Distributed

Sensor Networks

Journal of

Control Science

and Engineering
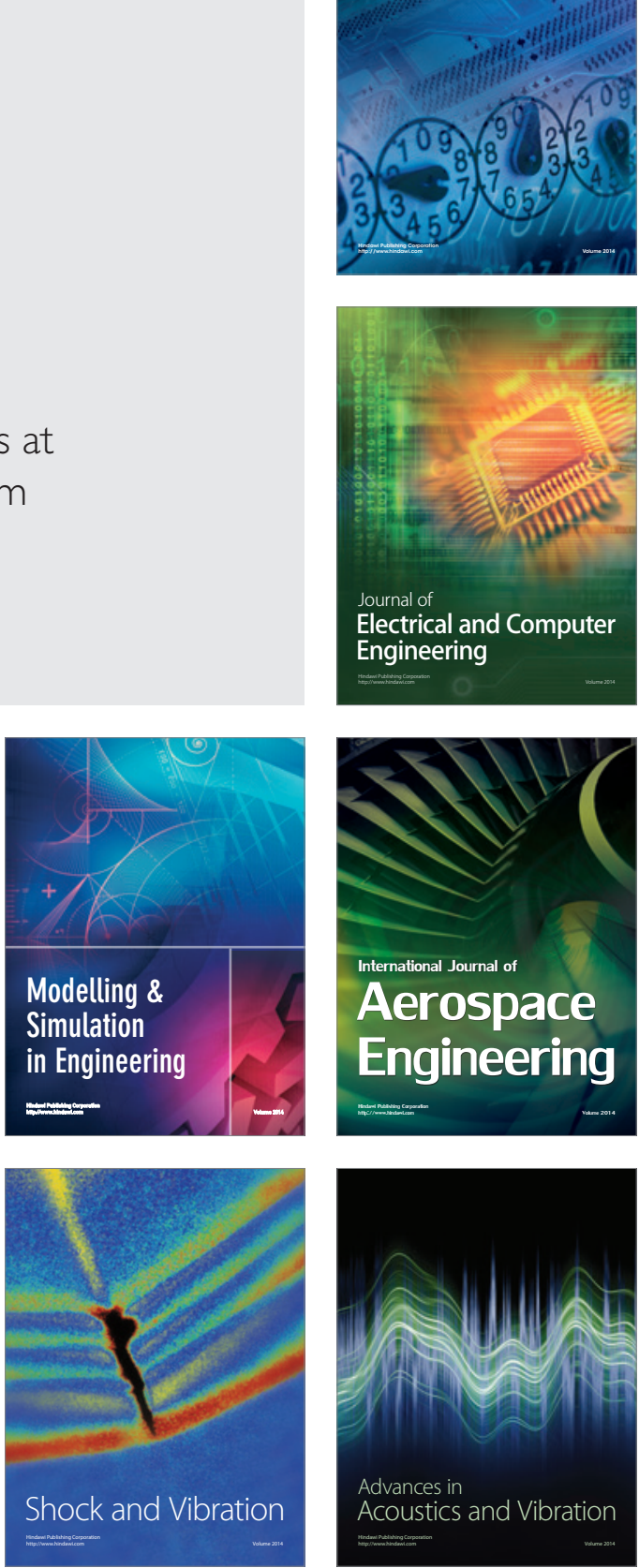